\title{
Positron Emission Tomography Studies of Cerebral Glucose Metabolism in Chronic Partial Epilepsy
}

\author{
Bassel W. Abou-Khalil, MD,* George J. Siegel, MD, ${ }^{*}$ J. Chris Sackellares, MD,* Sid Gilman, MD,* \\ Richard Hichwa, $\mathrm{PhD}, \dagger$ and Robert Marshall, MS*
}

Positron emission tomography (PET) performed with $\left[{ }^{18} \mathrm{~F}\right\}$-2-fluoro-2-deoxy-D-glucose ( $\left[{ }^{18} \mathrm{~F}\right\} \mathrm{FDG}$ ) was used to measure local cerebral metabolic rate for glucose (ICMRGIc) interictally in 31 patients with chronic partial epilepsy and 16 age-matched normal subjects. Hypometabolic zones were visualized in 25 patients (81\%). Cortical ICMRGlc in hypometabolic zones was within 2 standard deviations of the mean for normal temporal cortex in all but 8 patients. However, in 24 patients asymmetry between the hypometabolic cortex and homologous contralateral cortex was more than 2 standard deviations above the mean cortical asymmetry for normals. There was good correlation between hypometabolic zones and electroencephalogram (EEG) foci in patients with unilateral well-defined EEG foci. Diffuse or shifting EEG abnormalities were often associated with normal PET scans. Of 28 patients who underwent magnetic resonance imaging, 10 showed focal temporal lobe abnormalities corresponding to focal hypometabolism. While the $\left[{ }^{18}\right.$ F]FDG PET scan cannot currently localize an epileptogenic zone independently, the absence of focal hypometabolism or its presence contralateral to a presumed EEG focus suggests the need for additional electrophysiological data.

Abou-Khalil BW, Siegel GJ, Sackellares JC, Gilman S, Hichwa R, Marshall R: Positron emission tomography studies of cerebral glucose rnetabolism in chronic partial epilepsy. Ann Neuról 22:480-486, 1987

The classical view of epilepsy, stemming from the concepts of J. Hughlings Jackson [21], emphasizes cortical instability and discharges during seizures. More recent observations in studies of cerebral blood flow $[10,18]$, glucose metabolism $[11,18]$, electrophysiology [14], and behavior $[2,13]$ indicate that in epileptic patients brain function is altered interictally also.

Positron emission tomography (PET) with $\left[{ }^{18} \mathrm{~F}\right] 2$ fluoro-2-deoxy-D-glucose ( $\left.\left.{ }^{18} \mathrm{~F}\right] \mathrm{FDG}\right)$ has shown that, in the interictal state, local cerebral metabolic rate for glucose (ICMRGlc) is reduced in the region of the epileptogenic focus in 70 to $80 \%$ of patients with chronic partial epilepsy $[7,8,23]$. However, quantitative or standardized criteria for evaluaring the degree of hypometabolism and for comparing results from different studies have not been established. We used a standardized method to compare cortical asymmetry with absolute ICMRGlc and visual inspection of hypometabolic zones, and correlated the asymmetry with sites of surface electroencephalogram (EEG) abnormalities. We also compared the PET and EEG findings in patients with normal and abnormal magnetic reso- nance (MR) images. Finally, we assessed the clinical use of the PET data in the presence of conflicting or bilateral surface EEG abnormalities. A preliminary report of these results has been presented [1].

\section{Patients and Methods \\ Subjects}

Nineteen women and 12 men with partial seizures were studied. Their ages ranged from 16 to 72 years (mean 28 years); the age at seizure onset ranged from 2 months to 43 years (mean 11.5 years); and the duration of their epilepsy ranged from 3 to 30 years (mean 16.5 years). Seizure onset followed meningitis in 2 patients, encephalitis in 1 , significant head trauma in 2, and radiation therapy to the orbit for ocular tumor in 1. Nine patients had a history of childhood febrile convulsions, and 13 reported seizures in other family members. All patients had complex partial seizures, 23 had simple partial seizures, and 4 had tonic-clonic seizures.

Sixteen normal volunteers were studied, including 7 women and 9 men aged 18 to 53 years (mean 33 years). Informed consent was obtained. Subjects with progressive neurological disease or significant systemic or psychiatric disorders were excluded.
From the *Department of Neurology and $+P E T / C y c l o t r o n$ Facility, Division of Nuclear Medicine, Department of Internal Medicine, University of Michigan School of Medicine, Ann Arbor, MI 48109.

Received Sept 22, 1986, and in revised form Dec 15, 1986, and Feb 16, 1987. Accepted for publication Feb 19, 1987.
Address correspondence to Dr Siegel, Department of Neurology, Neuroscience Bldg, 1103 E Huron St, University of Michigan, Ann Arbor, MI 48109. 


\begin{tabular}{llllll}
\hline & \multicolumn{2}{c}{$\begin{array}{c}\text { Unilateral PET } \\
\text { Hypometabolic Zone }\end{array}$} & & & \\
\cline { 2 - 5 } & $\begin{array}{l}\text { Ipsilateral } \\
\text { to Dominant } \\
\text { EEG Focus }\end{array}$ & $\begin{array}{l}\text { Contralateral } \\
\text { to Dominant } \\
\text { EEG Focus }\end{array}$ & & $\begin{array}{l}\text { Bilateral PET } \\
\text { Hypometabolic } \\
\text { Zone }\end{array}$ & $\begin{array}{l}\text { No PET } \\
\text { Hypometabolic } \\
\text { Zone }\end{array}$ \\
\hline Unilateral Well-Defined Focus & 15 & 2 & 1 & 1 & $\ldots$ \\
Bilateral Well-Defined Foci & 3 & $\ldots$ & $\ldots$ & $\ldots$ & 5 \\
Shifting and Diffuse Abnormalities & $\ldots$ & 2 & $\ldots$ & $\ldots$ & $\ldots$ \\
\hline
\end{tabular}

EEG = electroencephalogram; PET $=$ positron emission tomography.

Table 2. Correlation of $M R, E E G$, and PET

\begin{tabular}{|c|c|c|c|}
\hline Patient ${ }^{\mathrm{a}}$ & $\mathrm{MR}^{\mathrm{b}}$ & $\mathrm{EEG}^{c}$ & PET $^{d}$ \\
\hline 1 & Left temporal & Left temporal & $\begin{array}{l}\text { Left lateral temporal; } \\
\text { Right mesial temporal }\end{array}$ \\
\hline 2 & Right temporal & $\begin{array}{l}\text { Bilateral independent; } \\
\text { Right temporal dominant }\end{array}$ & $\begin{array}{l}\text { Left anterior temporal; } \\
\text { Right posterior temporal }\end{array}$ \\
\hline 3 & Left temporal & $\begin{array}{l}\text { Bilateral independent; } \\
\text { Left temporal dominant }\end{array}$ & Left temporal-frontal \\
\hline 4 & Left temporal & Left temporal & Left temporal \\
\hline 5 & Left temporal & Diffuse and shifting & Left temporal \\
\hline $6^{e}$ & Left temporal & Left temporal & Left temporal \\
\hline 7 & Right temporal & Right temporal & Right temporal \\
\hline $8^{e}$ & Right temporal & Right temporal & Right temporal \\
\hline 9 & Left temporal & Left temporal & Left temporal \\
\hline 10 & Right temporal & Right temporal & Right temporal \\
\hline
\end{tabular}

a Patient 3 had a history of meningitis and patient 6 had prior radiation therapy to the left orbit. The other patients had no known etiological factors.

Location of high-intensity signal abnormality except for patient 1, who showed unilateral temporal lobe atrophy.

'EEG localization was based on interictal surface recordings in all the patients as in Table 1. Sphenoidal ictal recordings, where available, were nonlocalizing in patients 2 and 4 ; localizing to the left in patients 3,5 , and 6 ; and localizing to the right in patient 8 .

${ }^{\mathrm{L}}$ Location of relative hypometabolism.

eparients 6 and 8 had computed tomography abnormalities in the same region as the MR focus.

$\mathrm{MR}$ = magnetic resonance; $\mathrm{EEG}=$ electroencephalogram; PET = positron emission tomography.

Computed Tomography and Magnetic Resonance Scans All patients and normal volunteers underwent computed tomography (CT) scans. Twenty-eight patients (including 10 reported previously by Latack and associates [12]) were also subjected to MR imaging, which was performed with a 0.35 $T$ superconductive magnet utilizing a spin-echo pulse sequence. MR-signal abnormalities were interpreted as described previously [12]. In 29 patients the CT scans were normal or showed mild diffuse substance loss. Although a criterion for entry into the study was the absence of focal CT scan abnormalities, a subtle focal abnormality in the temporal lobe was found in 2 patients when their CT scans were visualized retrospectively after inspection of MR scans.

\section{$E E G$}

Scalp EEG recordings were obtained during PET studies on all patients and volunteers. Several interictal EEGs were recorded on all patients. Seizures in 18 patients were recorded on EEG and video. Fifteen patients underwent prolonged EEG monitoring with sphenoidal electrodes [16], and EEG recordings from chronically implanted bilateral depth electrodes were obtained in 2 of the 15.

On the basis of interictal surface EEGs (scalp and sphenoidal electrodes), patients were classified as having a unilateral well-defined focus, bilateral well-defined foci, or diffuse and shifting abnormalities (Table 1). One patient (Table 2, patient 6) who had normal interictal EEGs but frequent left temporal ictal discharges was classified as having a unilateral well-defined focus. Well-defined EEG foci were further classified as focal-temporal or parasagittal-temporal.

\section{PET Scans}

The instrumentation and procedures for the $\left[{ }^{18} \mathrm{~F}\right] \mathrm{FDG}$ PET scans have been described previously [9]. All patients underwent PET scans in the interictal state while maintained on their usual antiepileptic drugs. Subjects were scanned in a quiet, dim room; their eyes were covered but their ears were not plugged. Five to $10 \mathrm{mCi}$ of $\left[{ }^{18} \mathrm{~F}\right] \mathrm{FDG}$ was injected intravenously and radial arterial blood was sampled for $\left[{ }^{18} \mathrm{~F}\right\}$ plasma concentrations. Image planes were parallel to the 

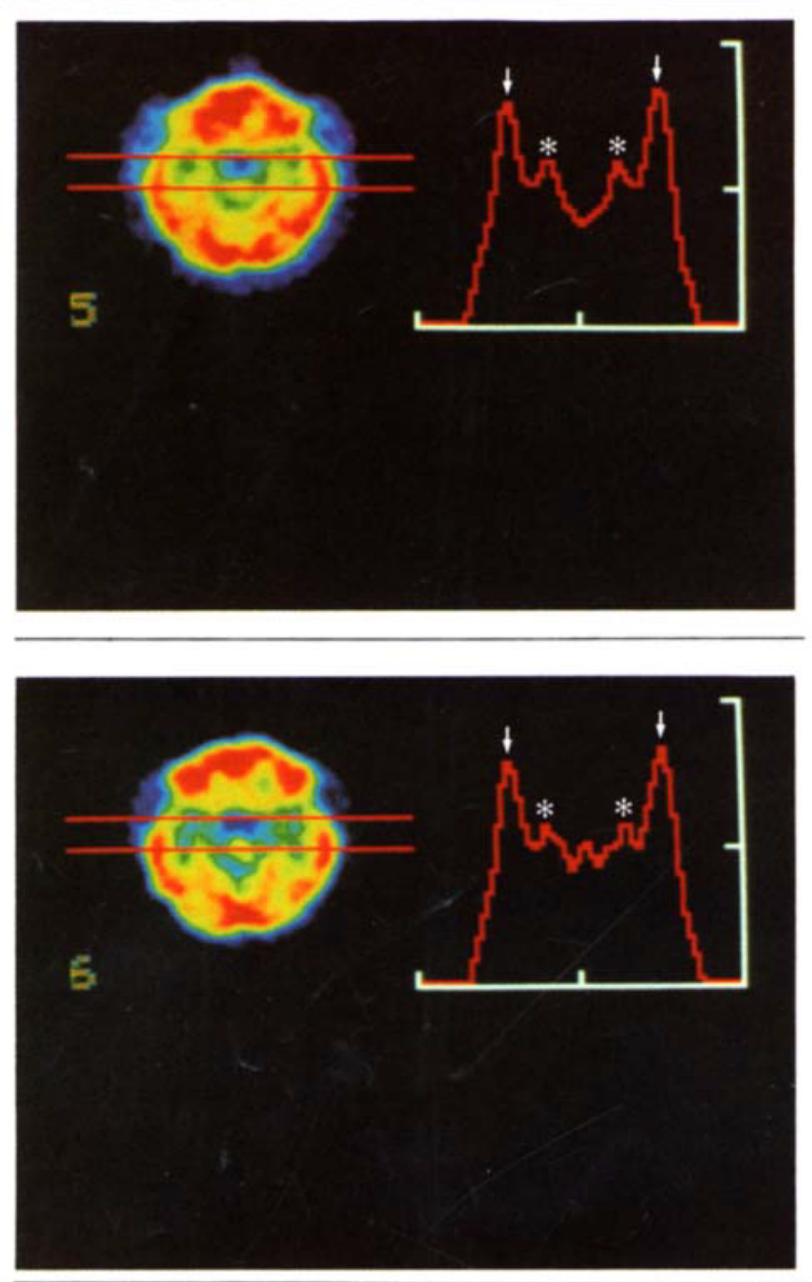

Fig 1. Two consecutive $\left({ }^{18} F\right\} 2-f$ fuoro-2-deoxy-D-glucose positron emission tomography scan levels in a normal subject. Horizontal sweeps for local cerebral metabolic rate for glucose in 8 rows of single pixels were made between the lines shown in the scan image (left). Single peak cortical pixels were identified in each of the 8 rows and used for calculation of cortical mean values in Table 3. The mean values for each vertical column containing 8 rows are plotted on the graph (right). The peaks in the graph represent lateral (arrows) and mesial (asterisks) temporal cortex.

canthomeatal line and started $2 \mathrm{~cm}$ above it. Ten slices 11 $\mathrm{mm}$ apart were obtained for 4 patients, and 15 slices for the remaining 29 patients. Ten lower slices $5.5 \mathrm{~mm}$ apart and 5 upper slices $11 \mathrm{~mm}$ apart were also taken. PET data were analyzed using standard single scan techniques for the calculation of the ICMRGlc parametric images as described previously [9] based on published methods $[15,19]$.

\section{PET Analysis}

PET scans were reviewed independently by two investigators, one of whom was blinded to the EEG data. Regions of relative hypometabolism were selected by visual inspection and considered significant only if there was evidence of hypometabolism on at least 2 levels. Peak cortical ICMRGlc was identified in single pixels on contiguous horizontal histograms of single rows of pixels through the image from anterior to posterior. Single pixels showing the highest values

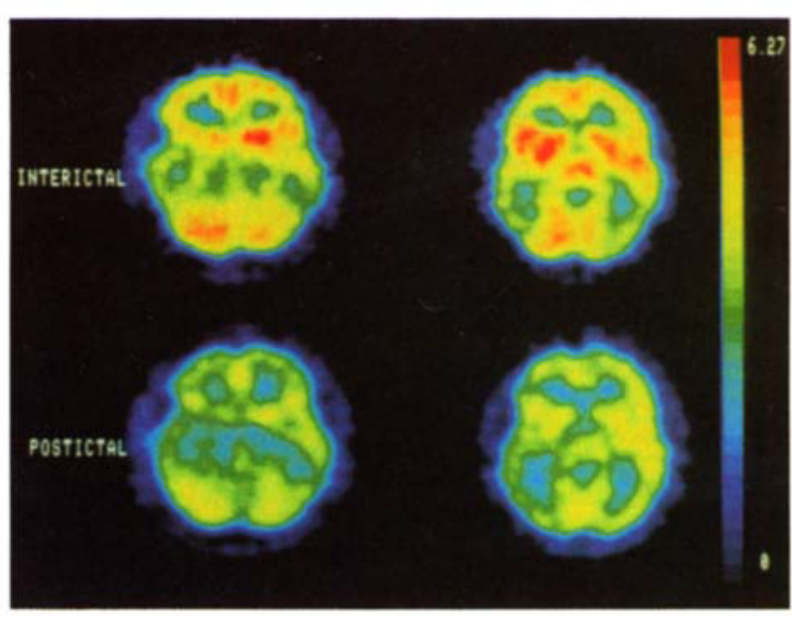

Fig 2. Interictal and postictal $\left({ }^{18} \mathrm{~F}\right) 2$-fluoro-2-deoxy-D-glucose $\left(\left(^{18} F\right\} F D G\right)$ positron emission tomography scans in a patient with partial seizures and secondary generalization. (Upper) Interictal scan showing bilateral bypometabolic zones in the left anterior and right posterior temporal regions. (Lower) Postictal scan obtained with $\left({ }^{18} \mathrm{~F}\right) \mathrm{FDG}$ injection 20 minutes after a secondarily generalized tonic-clonic seizure. In addition to a generalized decrease in local cerebral metabolic rate for glucose, there is widening of the left temporial hypometabolic zone as compared to the interictal scan.

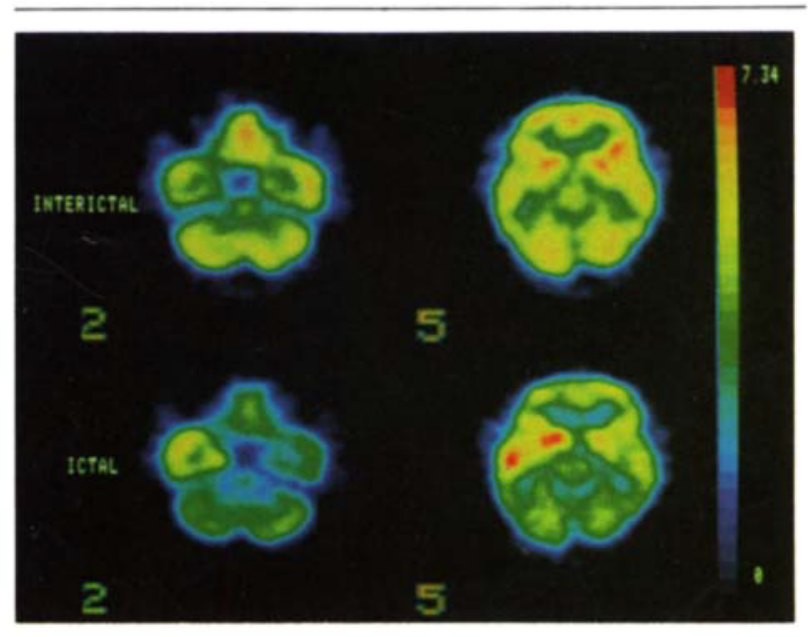

Fig 4. Interictal and ictall $\left.{ }^{18} \mathrm{~F}\right) 2$-fluoro-2-deoxy-D-glucose $\left(\left(^{18} F\right\} F D G\right)$ positron emission tomography (PET) scans in a patient with complex partial seizures. (Upper) Seizure occurred 11 minutes after $\left(^{18} \mathrm{FHFDG}\right.$ injection. Slight left temporal bypometabolism can be appreciated. (Lower) PET scan obtained with $\left({ }^{18} F\right) F D G$ injected seconds after the onset of a complex partial seizure. There is focal bypermetabolism in the left temporal lobe and caudate nucleus, and relative bypometabolism in the rest of the brain in these levels.

within the cortical regions were taken as peak cortical ICMRGlc. The peak cortical pixels for lateral and mesial temporal cortical regions could be easily identified on these histograms (Fig 1). The ICMRGIc was measured in the cortical peak within the nadir (cortical pixels with lowest ICMRGlc) of the hypometabolic zone and in the contralat- 
eral homotopic cortex. The ICMRGlc in the hypometabolic zone was compared to the contralateral side using the following asymmetry index $(\mathrm{AI}): \mathrm{AI}=(\mathrm{L}-\mathrm{R}) \times 100 /[(\mathrm{L}+\mathrm{R}) /$ 2]. In this calculation, $A I$ is the difference between the left (L) and right (R) ICMRGic pixel values expressed as a percentage of the mean of the pair $(L+R)$. A negative value for AI indicates that the left side ICMRGIc is lower than the right.

Both ICMRGlc and AI were calculated for lateral and mesial temporal cortical regions in the 16 normal volunteers using the standardized method described above. Normal mean temporal cortex values are based on calculations for 8 single peak cortical pixels from anterior to posterior in each of 2 consecutive image levels containing both mesial and lateral temporal cortex in the 16 subjects (see Fig 1). This was the most common location of hypometabolism in epileptic patients.

\section{Results}

PET Scans

Focal hypometabolism was found by visual inspection in 25 of 31 patients $(81 \%$ ) (see Table 1 ). The abnormalities were localized as follows: unilateral temporal in 17 patients, unilateral temporofrontal in 5 patients, and bilateral temporal in 3 patients. Selective frontal hypometabolism was not observed in these patients.

\section{Correlation of PET and EEG}

Table 1 shows that 15 of the 22 patients with unilateral hypometabolic zones $(68 \%)$ had a well-defined EEG focus ipsilateral to the zone of relative hypometabolism. Two of the 22 patients showed focal hypometabolism contralateral to a well-defined EEG focus. In these 2 patients, the EEG focus was located in the parasagittal-temporal electrode derivations, while EEG spikes in all other patients with a unilateral focus were confined mainly to the temporal regions. In contrast, only 1 of the 6 patients with no hypometabolic zone had a well-defined EEG focus. In this case, the EEG records showed only rare left sphenoidal sharp waves, while most other patients with well-localized EEG foci and regional hypometabolism had frequent epileptiform activity. In the remaining 5 patients with normal PET scans, the EEG showed shifting and diffuse abnormalities.

Three of the 5 patients with unilateral hypometabolism but bilateral interictal EEG foci or diffuse and shifting EEG abnormalities had one ictal focus demonstrated by sphenoidal or depth ictal recordings. In all 3 the ictal focus corresponded to the hypometabolic zone. Thus, a unilateral hypometabolic zone may indicate the side of ictal onset in the presence of bilateral interictal surface EEG abnormalities.

Five of the 7 patients with diffuse and shifting EEG abnormalities had normal PET scans (see Table 1). In 3 of the 5, multiple ictal recordings were available but not localizing. Two other patients with diffuse EEG changes had regional hypometabolism. One had ictal
Table 3. Peak Cortical lCMRGlc in Normal Volunteers ${ }^{\mathrm{a}}$

\begin{tabular}{lcr}
\hline & $\begin{array}{l}\text { Lateral Temporal } \\
\text { Cortex }\end{array}$ & \multicolumn{1}{l}{$\begin{array}{l}\text { Mesial Temporal } \\
\text { Cortex }\end{array}$} \\
\hline ICMRGIc Left $^{\mathrm{b}}$ & $5.99( \pm 1.22)$ & $4.69( \pm 1.00)$ \\
ICMRGIc $^{\mathrm{b}}$ Right & $6.09( \pm 1.28)$ & $4.65( \pm 0.95)$ \\
Asymmetry Index $^{c}$ & $-1.6 \%( \pm 10 \%)$ & $-0.5 \%( \pm 9 \%)$ \\
\hline
\end{tabular}

${ }^{a}$ All values are mean $( \pm S D) ; n=256$ data points from 16 subjects.

${ }^{b}$ Unit is $\mathrm{mg}$ glucose $/ \mathrm{min} / 100 \mathrm{~g}$ of brain tissue.

'Asymmetry index is calculated as described in Methods.

ICMRGlc = local cerebral metabolic rate for glucose.

recordings showing corresponding focal onset while the other had no ictal record available. Of 19 patients with unilateral and 5 with bilateral well-defined EEG foci, only 1 patient had a normal PET scan (see Table 1).

Only 2 of the 5 patients with bilateral well-defined EEG foci also had bilateral zones of hypometabolism (Fig 2). Multiple ictal recordings in these 2 patients were nonlocalizing.

\section{Correlation of EEG and PET Scans with MR Images}

Ten of the 28 patients scanned with MR had focal changes on MR images (see Table 2). The MR changes consisted of increased signal intensity, which was best seen on $T_{2}$-weighted images, in the temporal lobe in 9 patients and unilateral temporal lobe atrophy in $1 \mathrm{pa}$ tient. Seven of the 10 patients, including the one with substance loss, had unilateral well-defined surface EEG foci in the corresponding temporal lobe (see Table 2). Two patients had bilateral independent surface EEG foci with the dominant focus in the temporal lobe corresponding to the focal MR signal. In one of these 2 patients (see Table 2, patient 3), surface ictal recordings with sphenoidal electrodes confirmed a seizure focus corresponding anatomically to the focal MR and PET abnormalities. The remaining patient with focal MR signal abnormalities showed shifting surface EEG abnormalities. However, ictal recordings with sphenoidal electrodes available in that patient (see Table 2, patient 5) confirmed a mesial temporal seizure focus corresponding to the location of focal MR and PET abnormalities. Thus, all the patients with MR abnormalities had either an ictal focus or a single well-defined or dominant interictal focus in the corresponding temporal lobe.

All 10 patients with abnormal MR images showed regions of hypometabolism in the corresponding temporal lobes (see Table 2). In addition, 2 of these patients showed a second zone of hypometabolism in the opposite temporal lobe. One of these (see Table 2, patient 1), with left temporal lobe atrophy on $M R$, had a left unilateral interictal EEG focus, and one (see Table 2, patient 2) had bilateral interictal EEG foci with the dominant focus corresponding to the side of abnormal MR-signal. 


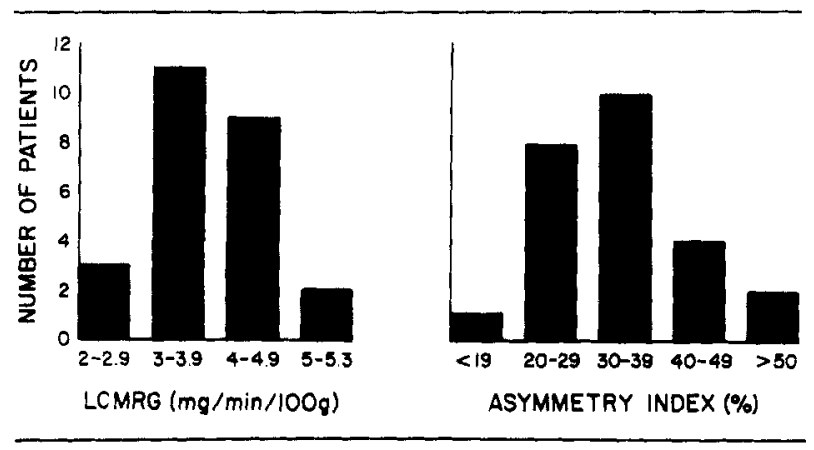

Fig 3. Hypometabolic regions in patients with partial seizures. Hypometabolic zones on $\left({ }^{18} \mathrm{~F}\right\} 2-f$ uoro-2-deoxy-D-glucose positron emission tomography (PET) scans were identified visually. Lateral temporal cortical peak local cerebral metabolic rate for glucose ( $L C M R G l c)$ in the nadir of the bypometabolic zone was measured and compared to the contralateral bomotopic cortex (asymmetry index (AIf) as described in Metbods. For the 3 patients whose PET scans were judged to bave bilateral bypometabolic zones, only the zone with largest $A I$ in each is included in this figure. (Left panel) Lateral temporal cortical peak ICMRGlc in nadir of bypometabolic zones. (Right panel) Asymmetry index of bypometabolic zones. (Compare with normal values in Table 3.)

Normal MR imaging was obtained on 11 patients with unilateral hypometabolism, 1 with bilateral hypometabolism (and bilateral independent EEG foci), and 6 with normal PET scans.

\section{Comparison of AI with Absolute ICMRGlc Values}

Normative data for cortical peak ICMRGlc are summarized in Table 3. Note the wider range of variability in absolute ICMRGlc than in AI. The standard deviation (SD) for absolute ICMRGlc values in lateral and mesial temporal cortex is 20 to $21 \%$ of the means, while the SD for AI is $10 \%$ of the means of the homotopic pairs in normal subjects (see Table 3). In addition, the ICMRGlc in mesial temporal cortex is about $13 \%$ less than in lateral temporal cortex (see Table 3).

Figure 3 shows ICMRGic and AI values for the 25 patients whose lateral temporal hypometabolic regions were identified visually. Absolute cortical ICMRGlc values in the nadir of the hypometabolic zones in 17 of the 25 patients were within 2 SDs of the normal mean. In contrast, the AI was outside $2 \mathrm{SDs}$ of the normal mean in all but one case (see Fig 3). Accordingly, the relative $A I$ values are much more sensitive than the absolute ICMRGic values in distinguishing epileptic patients from normal subjects.

\section{Correlation of PET Findings with Clinical Data}

No significant relationship was found between the presence of a hypometabolic zone and the following clinical characteristics: age, sex, age at seizure onset, duration of epilepsy, seizure frequency, type of partial seizure, family history of epilepsy, and history of febrile convulsions. However, all 6 patients with an identified etiologic factor in their history had an abnormal PET scan.

\section{Postictal PET Scans}

In addition to their interictal scans, postictal PET scans were obtained in 2 patients. In both, $\left[{ }^{18} \mathrm{~F}\right] \mathrm{FDG}$ was injected 20 minutes after a complex partial seizure with secondary generalization. In the first patient, the interictal scan was normal but the postictal scan showed a maximum of $28 \%$ asymmetry in the temporal and occipital cortex. In that patient, neither the interictal nor the ictal EEG permitted localization of the focus. In the second patient, whose interictal scan showed a maximum. anterior temporal cortex asymmetry of $17 \%$, the hypometabolic region widened in area and the maximum anterior lateral temporal cortex asymmetry increased to $24 \%$ in the postictal scan (see Fig 2). In this case, the interictal EEG showed bilateral independent temporal lobe foci, and the ictal EEG did not permit localization of the seizure onset. These data indicate that seizures may produce transient hypometabolism postictally.

\section{Ictal PET Scans}

Two ictal scans were obtained. In one $\left[{ }^{18}\right.$ F]FDG was injected within seconds after the onset of a complex partial seizure. In the second, $\left[{ }^{18} \mathrm{~F}\right] \mathrm{FDG}$ was injected within 10 seconds of the onset of loss of consciousness, which followed a 2-minute aura. In the first case, a well-defined area of relative hypermetabolism was seen in one temporal lobe, corresponding to a previously observed interictal hypometabolic zone (Fig 4). This region also corresponded to the EEG focus. It is of interest that the relative hypermetabolism extends to the ipsilateral caudate nucleus (see Fig 4). In the second case (not shown), ICMRGlc was increased asymmetrically in both temporal lobes compared to the interictal scan. The interictal PET scan, however, was symmetrical, and localization of an EEG focus was not possible on interictal or ictal recordings.

\section{Discussion \\ PET Scan Analysis}

Our data show that in the temporal cortex, which is the most common site of hypometabolism, there is normally a large variability in ICMRGlc (see Table 3). Many of the visually detected hypometabolic zones do not correspond with absolute ICMRGlc values outside of the normal range (see Fig 3). However, all but one of the visually detected hypometabolic regions correspond with AI values of $20 \%$ or more (see Fig 3). 
Thus, using an arbitrary cutoff of 2 SDs (20\%, see Table 3), the AI value permitted identification of an abnormal PET scan in 24 of 31 epileptic patients $(77 \%)$. Basing the analysis on comparison of the cortical peaks within the suspected region and the homologous contralateral region (AI) provides a standard method that allows comparisons between scans or among studies. However, the method does not completely eliminate investigator bias, since only pixels within the visually discerned hypometabolic zone were analyzed.

\section{Correlation of PET and EEG}

Patients with a well-defined unilateral EEG focus tended to have PET scans that showed a single ipsilateral hypometabolic zone (see Table 1). In contrast, patients with the least localizing EEGs were most likely to have a normal PET scan (see Table 1).

Three of the 5 parients with bilateral well-defined interictal surface EEG foci had only unilateral zones of hypometabolism (see Table 1), but ictal onset data in 2 of the 3 correlated with the side of hypometabolism. In some cases, one hemisphere may contain a mirror focus. Hypometabolism may not always be produced in mirror foci of secondary epileptogenesis. Alternatively, the wide range of normal variation in 1CMRGlc and reliance on asymmetry measurements may cause investigators to miss contralateral changes.

An important question is how to use the $\left[{ }^{18} \mathrm{~F}\right] \mathrm{FDG}$ PET data in determining the side of epileptogenic foci. It is evident from Table 1 and earlier studies $[3,7,8$, 23] that when there is a well-defined unilateral EEG focus, the $\left[{ }^{18} \mathrm{~F}\right] \mathrm{FDG}$ PET scan is likely to show an ipsilateral hypometabolic region. Thus, the $\left[{ }^{18} \mathrm{~F}\right] \mathrm{FDG}$ PET scan, when positive in such patients, confirms the EEG data and may eliminate the need for additional confirmation from implanted depth electrodes.

However, cases where a new means of establishing the epileptogenic focus is most needed are those without well-defined unilateral EEG abnormalities on scalp and sphenoidal recordings. In these cases, the $\left[{ }^{18} \mathrm{~F}\right] \mathrm{FDG}$ PET scan does not provide the data necessary to identify an epileptogenic source. The $\left[{ }^{18} \mathrm{~F}\right] \mathrm{FDG}$ PET scan is often normal in the presence of diffuse or shifting EEG abnormalities (see Table 1) and nonlocalizing ictal recordings. Hypometabolism by itself is nonspecific with regard to tissue pathophysiology and does not establish epileptogeniciry. In patients with a hypometabolic zone but no corresponding welldefined EEG focus, additional EEG studies, such as depth electrode recordings, may be required.

We wished to evaluate the significance of focal hypometabolism in the presence of interictal surface EEG data that conflict with PET results. Our experience with available ictal data from depth electrodes in 1 patient and from sphenoidal electrodes in 2 patients supports the conclusion that in the presence of bilateral interictal surface EEG epileptiform abnormalities, focal hypometabolism indicates the side of ictal onset [6]. Therefore, if the hypometabolic region does not agree with an otherwise well-defined surface EEG focus, additional data, such as depth electrode recordings, should be obtained to localize the epileptogenic focus.

\section{Correlation of PET and MR Scans}

Foci of increased MR signal in the temporal lobe are found in approximately a third of patients with partial seizures in this series (see Table 2). When present, increased MR signal is correlated with an EEG focus and region of hypometabolism in the same temporal lobe (see Table 2). However, caution is necessary. In one series of patients, an MR signal abnormality was observed contralateral to an EEG focus and hypometabolism [22]. In another series, 3 patients with focal abnormal MR signal had normal $\left[{ }^{18} \mathrm{~F}\right] \mathrm{FDG}$ PET scans $[20]$.

\section{Dynamic Properties of the Hypometabolic Zone}

The pattern of hypometabolism is not fixed, but appears to change in relation to ictal events. The two PET scans repeated 20 minutes postictally after secondarily generalized seizures showed the appearance of new areas of hypometabolism in one, and intensification and wider encroachment of hypometabolism in the other (see Fig 2). The 2 ictal $\left[{ }^{18}\right.$ F]FDG PET scans were very different in appearance. One showed focal hypermetabolism corresponding to the seizure focus, while the other scan was nonlocalizing. There are very few observations concerning subcortical structures in patients during partial seizures [4]. Involvement of the thalamus has been observed in one case [5]. Figure 4 shows that the ipsilateral caudate nucleus may be involved in a partial complex seizure.

The 30-minute uptake period in $\left[{ }^{18} \mathrm{~F}\right] \mathrm{FDG}$ PET studies limits their usefulness in kinetic studies. Further investigation with tracers such as oxygen- $\mathrm{O}^{15}$ allowing repeated studies with short intervals are needed to elucidate the clinical significance of ictal and postictal scans [17].

This research was supported by NIH grant NS15655. The authors are grateful for the assistance of R. Ehrenkaufer, J. Rothley, A Tornow, and $\mathrm{L}$. Weinberger in the performance of this work, and for the support of W. Beierwaltes, retired chief of the Division of Nuclear Medicine.

\section{References}

1. Abou-Khalil BW, Sackellares JC, Hichwa RD, et al: Local cerebral metabolic rate for glucose (LCMRG) in patients with chronic refractory partial seizures. In Wolf $\mathrm{P}$, Dam $\mathrm{M}$, Dieter J, Dreifuss FE (eds): Advances in Epileptology, XVIth International Symposium. New York, Raven, 1987, pp 143-145 
2. Berent S, Sackellares JC, Abou-Khalil BW, et al: PET studies of cerebral giucose metabolic activity in temporal lobe epilepsy: the functional implications of lateralized hypometabolism. Neurology 36(Suppl):337, 1986

3. Engel J Jr, Brown WJ, Kuhl DE, et al: Pathological findings underlying focal temporal lobe hypometabolism in partial epilepsy. Ann Neurol 12:518-528, 1982

4. Engel J Jr, Kuhl DE, Phelps ME: Patterns of human local cerebral glucose metabolism during epileptic seizures. Science 218:64-66, 1982

5. Engel J Jr, Kuhl DE, Phelps ME: Regional brain metabolism during seizures in humans. Adv Neurol 34:141-148, 1983

6. Engel J Jr, Kuhl DE, Phelps ME, Crandall PH: Comparative localization of epileptic foci in partial epilepsy by PCT and EEG. Ann Neurol 12:529-537, 1982

7. Engel J Jr, Kuhl DE, Phelps ME, Mazziotta JC: Interictal cerebral glucose metabolism in partial epilepsy and its relation to EEG changes. Ann Neurol 12:510-517, 1982

8. Gloor P, Yamamoto L, Ochs R, et al: Regional cerebral metabolism measured by positron emission tomography in patients with partial epilepsy: correlation with EEG findings. In Porter RJ, et al (eds): Advances in epileptology: XVth epilepsy international symposium. New York, Raven, 1984, pp 99-103

9. Hichwa RD: Positron production and PET scanning. IEEE Trans Nucl Sci NS-30:1688-1692, 1983

10. Ingvar $\mathrm{DH}$ : rCBF in focal cortical epilepsy. In Langfitt $T W$, et al (eds): Cerebral circulation and metabolism. Berlin, SpringerVerlag, 1975, pp 361-364

11. Kuhi DE, Engel J Jr, Phelps ME, Selin C: Epileptic patterns of local cerebral metabolism and perfusion in humans determined by emission computed tomography of ${ }^{18} \mathrm{FDG}$ and ${ }^{13} \mathrm{NH}_{3}$. Ann Neurol 8:348-360, 1980

12. Latack JT, Abou-Khalil BW, Siegel GJ, et al: Patients with partial seizures: evaluation by MR, CT, and PET imaging. Radiology 159:159-163, 1986
13. Mayeux R, Brandt J, Rosen J, Benson DF: Interictal memory and language impairment in temporal lobe epilepsy. Neurology 30:120-125, 1980

14. Pedley TA: Epilepsy and the human electroencephalogram. In Schwartzkroin PA, W/heals HV (eds): Electrophysiology of Epilepsy. London, Academic, 1984, pp 1-30

15. Phelps ME, Huang SC, Hoffman EJ, et al: Tomographic measurement of local cerebral glucose metabolic rate in humans with (F-18)2-fluoro-2-deoxy-D-glucose: validation of method Ann Neurol 6:371-388, 1979

16. Rovit RL, Gloor P, Henderson LR: Temporal lobe epilepsy-a study using multiple basal electrodes. I. Description of method. Neurochirurgia 3:5-18, 1960

17. Sackellares JC, Abou-Khalil BW, Siegel GJ, et al: PET studies of interictal, ictal and postictal changes in local cerebral blood flow in temporal lobe epilepsy. Neurology 36(Suppl 1):338, 1986

18. Siegel GJ, Abou-Khalil BW, Sackellares JC: Imaging of regional cerebral metabolism and blood flow in epilepsy. In Sen AK, Lee $\mathrm{T}$ (eds): Brain Receptors in Psychiatry and Neurology. Cambridge, UK: Cambridge University Press (in press)

19. Sokoloff $\mathrm{L}$ : The relationships between function and energy metabolism: its use in the localization of functional activity in the nervous system. Neurosci Res Program Bull 19:159-210, 1981

20. Sperling MR, Wilson G, Engel J Jr, et al: Magnetic resonance imaging in intractable partial epilepsy: correlative studies. Ann Neurol 20:57-62, 1986

21. Taylor J (ed): Selected Writings of John Hughlings Jackson. Vol I. New York, Basic, 1958, pp 94-96, 100, 279

22. Theodore $\mathbf{W H}$, Dorwart $\mathbf{R}$, Holmes $M$, et al: Neuroimaging in refractory partial seizures: comparison of PET, CT, and MRI. Neurology 36:750-759, 1986

23. Theodore WH, Newmark ME, Sato S, et al: 18 F Fluorodeoxyglucose positron emission tomography in refractory complex partial seizures. Ann Neurol 14:429-443, 1983 